\title{
Publisher Correction: The gut-liver axis and the intersection with the microbiome
}

Anupriya Tripathi, Justine Debelius, David A. Brenner, Michael Karin, Rohit Loomba, Bernd Schnabl and Rob Knight $(\mathbb{D}$

Nature Reviews Gastroenterology \& Hepatology https://doi.org/10.1038/s41575-018-0011-z (2018) Published online 10 May 2018

In the original version of Table 1 published online, upward arrows to indicate increased translocation of PAMPs were missing from the row entitled 'Translocation' for both the column on alcoholic liver disease and nonalcoholic fatty liver disease. This error has now been updated in the PDF and HTML version of the article.

https://doi.org/10.1038/s41575-018-0031-8 I Published online 21 May 2018 